\title{
GESTATIONAL GIGANTOMASTIA
}

Letícia Augusto Garcia

${ }^{1}$ Hospital Guilherme Álvaro - Santos (SP), Brazil.

Introduction: Gigantomastia is a disease of unknown etiology, which can occur in two different situations: gestational and non-gestational phases. The gestational type is a rare complication that affects 1:28,000 to 1:100,000 pregnancies and is usually bilateral. It consists of a diffuse, massive breast increase during pregnancy. In a normal pregnancy, the breasts double in size without sequelae; however, in this disease, the breast tissue may experience a 10 to 20 -fold increase. The treatment varies from drug therapy to plastic surgery (mammaplasty) and radical surgery (mastectomy). Objectives: To report a case of gestational gigantomastia, its treatment approach, and outcome. Methods: Data from this study were obtained from a multidisciplinary clinical experience, image records, and literature search. Case Report: An 18-year-old patient, admitted to the mastology department of Hospital Guilherme Álvaro, in Santos, Southeastern Brazil, was diagnosed with gigantomastia in her $23^{\text {rd }}$ week of pregnancy. The exacerbated breast growth during the gestational period caused pain and functional impairment to the patient. Bilateral mastectomy was performed at 27 weeks of pregnancy due to the worsening of her pulmonary condition, leading to the risk of maternal and fetal death. Macroscopic anatomopathological examination revealed breasts with a total weight of $27 \mathrm{~kg}$, lobular hyperplasia, pronounced stromal hyperplasia, and necrosis of the breast parenchyma. She stayed in the intensive care unit during the postoperative period with good progression. The patient had a normal delivery at 38 weeks of gestation with a live fetus. Discussion: The ideal management for gestational gigantomastia is not clear. The treatment includes surgeries (reduction mammaplasty and total mastectomy with or without reconstruction), medications, or a combination of both. In the current case, the treatment chosen was mastectomy during pregnancy due to respiratory distress and the risk of sepsis by ischemic tissue necrosis. During the surgical procedure, there was a risk of hemodynamic instability, given the large volume of breast removed, representing $40 \%$ of the total weight of the patient. Conclusion: Gestational gigantomastia is an exceptionally rare condition, and the literature has few reports on the subject. The therapeutic management depends on factors intrinsic to the patient, so each case requires individualization. The therapeutic decision aims at the best prognosis, taking into account possible complications and a reduction in maternal and fetal morbidity. 